\title{
Improving Histogram-Based Image Registration in Video Sequences through Warping
}

\author{
Xuesong Le, Ruben Gonzalez \\ School of Information, communication, and Technology, Griffith University \\ Griffith University, Parklands Drive, Southport QLD 4222 Australia
}

\begin{abstract}
This paper presents two computationally efficient dynamic-time warping algorithms for image registration in video sequence through histogram based image segmentation. The key idea is to warp the histogram in an input frame to create an approximation of a reference frame. Any histogram based thresholding method can then be applied to create consistent regions in both the input and the approximated reference frames. Experiments of the proposed algorithm are used to demonstrate that more consistent matches can found after thresholding.
\end{abstract}

Keywords: histogram-based; dynamic-time warping; consistent regions.

\section{Introduction}

Compared to numerous feature point based algorithms surveyed in [1-3], the use of image segmentation as a step in image registration for video sequences has been little explored due to the computational requirements and the difficulty in obtaining segmentation consistency. Flusser and Dai [4-5] proposed to extract closed regions by contour tracing detected edges. Goshtasby [6] iteratively split an image into regions recursively until the variation of gray-level histogram in each sub-region is small. These region-based segmentation methods are not widely used in image registration of video sequences due to the computational complexity in part created by the postprocessing requirements such as edge linking or boundary closing. In contrast, regionbased segmentation methods, such as histogram splitting methods are very efficient because they require only one pass through the pixels in the image to perform segmentation. The key parameter in the histogram thresholding process is the choice of the threshold values.

Histogram threshold selection algorithms can be categorized into two groups: shape-based and clustering-based. The first category of methods achieves thresholding based on the shape properties of the histogram. It comprises three stages: recognizing the modes of the histogram, finding the valleys between the identified modes and finally assigning the valleys as the optimal thresholds. One disadvantage of the mode-seeking method is that it may be difficult to identify significant peaks and valleys in the image. Rosenfeld et al. [7] proposed a valley sharpening technique which restricts the histogram to the pixels with large absolute values of derivatives. Cheng and Sun [8] proposed a peak finding algorithm to locate the globally 
significant peaks of the histogram by removing small peaks, peaks which are too close to adjacent peaks, and peaks if valleys between them are not obvious. In the class of clustering methods, gray levels are grouped under various clustering criteria. Otsu [9] selected the global optimal threshold by maximizing the weighted betweenclass variance or minimizing the weighted within-class variance. Kapur [10] considered a multi-level thresholding scheme where the sum of between-class entropies for two classes is maximized.

Recently, Goncalves [11] proposed an image registration method that uses histogram-based image segmentation but it can only handle translation and rotation because it is not robust to histogram changes and image scaling causes more significant change of the histogram. In this paper, we present a novel method that combines both dynamic time warping (DTW) methods and histogram thresholding techniques to solve this problem. The objective of warping two histograms is to minimize the histogram differences across frames, and thus provide higher correlation between images and creates increased segmentation consistency. In our method, the histogram of the second image is warped first to create the approximation of the histogram in the first image before histogram thresholding. Two efficient DTW algorithms are proposed here to align the histogram sequences. The direct consequence is that each gray level in the second histogram is mapped to its correspondent in the second histogram. Then histogram thresholding techniques in [710] can be used to find optimal thresholds for the first histogram. After that, the same thresholds with a lookup table created during the warping can be applied on the second histogram. Finally, regions in both images are found by applying the thresholds. The significance of proposed histogram warping operation is that more consistent regions are found, which reduces the number of outliers in image registration and improves the registration accuracy.

\section{Dynamic Time Warping in Histogram Alignment}

DTW is a technique that finds the optimal alignment between two time series if one time series is warped non-linearly by stretching or shrinking it along its time axis. This warping operation between two time series can then be used to find corresponding regions between the two time series. In our case, we are dealing with histograms rather than time series and so the DTW problem is expressed as follows: Given two histogram sequences F, and G, both of length 256,

$$
F=f_{0}, f_{1}, \ldots, f_{i}, \ldots f_{255} \square \square=g_{0}, g_{1}, \ldots, g_{j}, \ldots g_{255}
$$

Construct a warp path $\mathrm{W}$ :

$$
W=w_{0}, w_{1}, \ldots, w_{k}, \ldots w_{n}
$$

where $\mathrm{n}$ is the length of the warp path and the $\mathrm{k}^{\text {th }}$ element of the warp path is: 


$$
w_{k}=(i, j)
$$

where $\mathrm{i}$ is an index of histogram sequence $\mathrm{F}$, and $\mathrm{j}$ is an index of histogram sequence $G$. The warp path starts at the beginning of each histogram sequence at $\mathrm{w}_{0}=(0,0)$ and finishes at the end of both sequence at $\mathrm{w}_{\mathrm{n}}=(255,255)$. An example of warping histogram $F$ to another histogram $G$ is shown in Figure 1. If both of the histograms were identical, the warp path is a straight line joining $\mathrm{w}_{0}$ ad $\mathrm{w}_{\mathrm{n}}$. There is a constraint on the warp path that forces $i$ and $j$ to be monotonically increasing in the warp path.

$$
w_{k}=(i, j), w_{k+1}=\left(i^{\prime}, j^{\prime}\right), i \leq i^{\prime} \leq i+1, j \leq j^{\prime} \leq j+1
$$

The minimum distance path, $\mathrm{W}$, between $\mathrm{F}$ and $\mathrm{G}$ is defined as:

$$
\operatorname{Dist}(W)=\min \left(\sum_{k=0}^{k=n} \operatorname{Dist}\left(w_{k i}, w_{k j}\right)\right)
$$

where Dist $\left(\mathrm{w}_{\mathrm{ki}}, \mathrm{w}_{\mathrm{kj}}\right)$ can be any distance metric between two data point indexes (one from $\mathrm{F}$ and another from $\mathrm{G}$ ) in the $\mathrm{k}^{\text {th }}$ element of the warp path.

\subsection{Dynamic Programming Based DTW}

A direct solution to find the optimal warp path in equation 5 is through dynamic programming (DP). A 256x256 two dimension cost function matrix D in figure 1, is constructed first where each element, denoted as $D(i, j)$, is defined in equation 6 . Each $D(i, j)$ is the sum of the distance $d(i, j)$ found in the current cell and the minimum of the adjacent elements.

$$
D(i, j)=\left\{\begin{array}{cc}
\min [D(i, j-1), D(i-1, j), D(i-1, j-1)]+d(i, j) & \text { if }(i>0, \wedge j>0) \\
d(i, j) & \text { else }
\end{array}\right.
$$

where $d(i, j)=|f(i)-g(j)|$.

After the cost matrix D is filled, backtracking along the minimum cost index pairs $(\mathrm{i}, \mathrm{j})_{\mathrm{k}}$ starting from $(255,255)_{0}$ to $(0,0)_{\mathrm{n}}$ yields the DTW warping path W. A greedy search is performed that evaluates cells to the left, up, and diagonally to the top-left. Figure 1 below shows an example of a cost matrix and minimum-distance warp path traced through it from $\mathrm{D}(0,0)$ to $\mathrm{D}(255,255)$. If the warp path passes through a cell $D(i, j)$ in the cost matrix, it means that $i^{\text {th }}$ point in histogram $F$ is warped to the $j^{\text {th }}$ point in another histogram G.

The major disadvantage of the DP-based DTW is that it requires high complexity of $\mathrm{O}\left(\mathrm{N}^{2}\right)$. A number of complexity reduction methods [12] have been proposed to speed up by a large constant factor, but those methods still runs in $\mathrm{O}\left(\mathrm{N}^{2}\right)$ time. To avoid the brute-force dynamic programming, a fast approximation of the optimal 
solution is proposed in section 2.2 which directly aligns two histogram sequences in $\mathrm{O}(\mathrm{N})$ time.

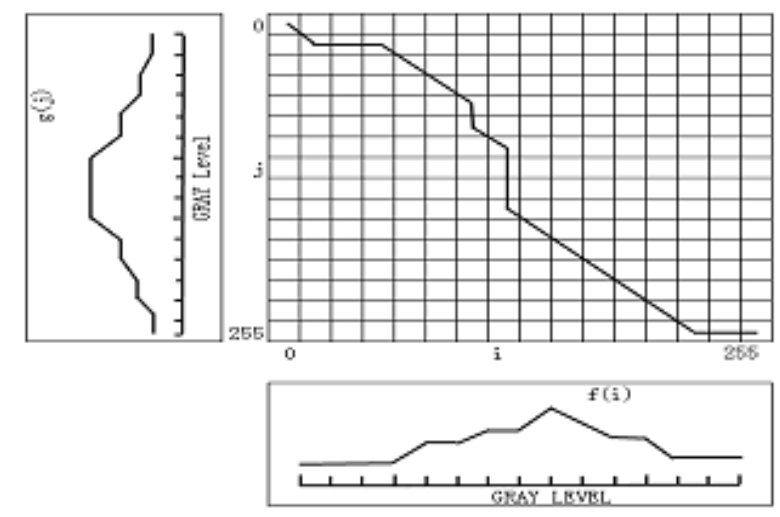

Figure 1. Illustration of a warping path between histogram F and G.

\subsection{Integration Based DTW}

A faster DTW approach is to use integration. In this approach, the histogram warping problem is described as follows: each gray level $i$ of the second histogram sequence, $\mathrm{G}$, is mapped into a new gray level $\mathrm{j}$ in the first histogram sequence, $\mathrm{F}$, such that the quantity:

$$
\begin{gathered}
\mid \sum_{k=0}^{j} c F[k]-\sum_{k=0}^{i} c G[k], \\
\sum_{k=0}^{i} c F[k]=\sum_{k=0}^{i-1} c F[k]+f[i] \sum_{k=0}^{i} c G[k]=\sum_{k=0}^{i-1} c G[k]+g[i]-
\end{gathered}
$$

is minimized subject to $\mathrm{j}$.

The mapping table can be constructed by a linear algorithm $(\mathrm{O}(\mathrm{N}))$ which does numerical integrations simultaneously on both histogram sequences. For each gray level $i$ in histogram $G$, we integrate histogram $F$ until a $j$ is reached such that the above condition is satisfied. The integration process exploits the simple recurrence relations as i increases from one to the other. Thus, the overall cost in constructing the mapping table is linear in time.

The C code of warping histogram sequence $G$ with reference to $F$ is essentially a few lines of code:

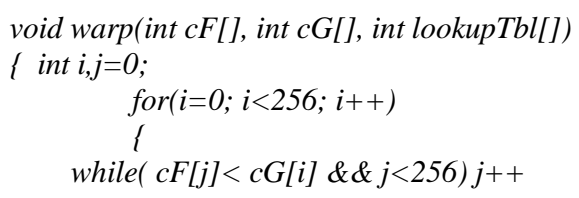




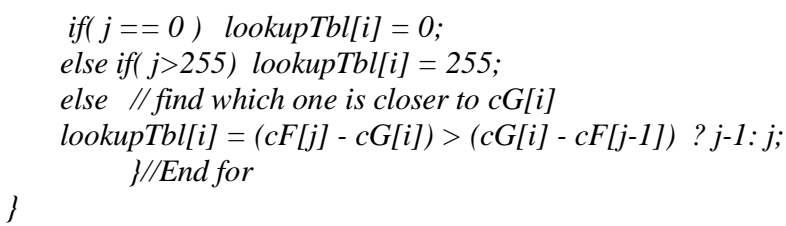

Table 1 lists the average running time on a 600x400 video sequence between DPwarped method and integration-warped method. Experiment runs on a PC with 3.00GHZ Intel Pentium 4 CPU and 1G Memory.

\begin{tabular}{|l|c|c|}
\hline & Integration- based DTW & DP-based DTW \\
\hline comp. time (ms) & 0.0287 & 12.5309 \\
\hline
\end{tabular}

Table 1 Running Time Comparison between Integration-Based DTW and DP-Based DTW

In the above experiment, the DP-based DTW, is 430 times slower than the integration-based method shown in Table 1 since the DP-based DTW runs in $\mathrm{O}\left(\mathrm{N}^{2}\right)$ time while integration-based method runs in $\mathrm{O}(\mathrm{N})$.

\section{Proposed Histogram Thresholding Algorithms}

After the mapping of gray levels in both histograms is established, a histogram thresholding technique is still required to select optimal levels for the histogram in the first image. We propose three representative thresholding algorithms here and use them during experiments. Two of them are extensions of existing methods.

The problem of multi-level histogram thresholding can be stated as following: It assumes that there are $M-1$ thresholds, $\left\{t_{1}, t_{2}, \ldots, t_{M-1}\right\}$, which divide the original histogram into $M$ classes: $C_{1}$ for $\left[0, \ldots, t_{1}\right], C_{2}$ for $\left[t_{1}+1, \ldots, t_{2}\right], \ldots, C_{i}$ for $\left[t_{i-1}+1, \ldots\right.$, $\left.\mathrm{t}_{\mathrm{i}}\right], \ldots$, and $\mathrm{C}_{\mathrm{M}}$ for $\left[\mathrm{t}_{\mathrm{M}-1}+1, \ldots, 255\right]$.

\subsection{Clustering-Based Thresholding}

The proposed clustering algorithm is an extension of OTSU's bi-level thresholding method [9] to a multi-level thresholding method. The cumulative probabilities, $\omega_{k}$, mean, $\mu_{k}$, and total variance, $\sigma_{k}^{2}$, mean for each class $\mathrm{C}_{\mathrm{k}}$ is defined as:

$\omega_{k}=\sum_{i=C_{k}} p_{i} \mu_{k}=\sum_{i=C_{k}} i p_{i} / \omega_{k} \quad \sigma_{k}^{2}=\sum_{i=C_{k}}\left(i-\mu_{k}\right)^{2} p_{i} / \omega_{k}$

In [11], the optimal threshold $t$ in any class $C_{i}$ for $\left[t_{i-1}+1, \ldots, t_{i}\right]$ is the maximum weighted between-class variance, $\sigma_{B}^{2}$ :

$$
\sigma_{B}^{2}=\omega_{1} \omega_{2}\left(\mu_{1}-\mu_{2}\right)^{2}
$$

$\omega \eta \varepsilon \rho \varepsilon:$

$\omega_{1}=\sum_{j=t_{i-1}}^{t} p_{j}, \omega_{2}=\sum_{j=t+1}^{t_{i}} p_{j}, \mu_{1}=\sum_{j=t_{i-1}}^{t} j p_{j} / \omega_{1}, \alpha \nu \delta \delta_{2}=\sum_{j=t+1}^{t_{i}} j p_{j} / \omega_{2}$. 
For this work, we have instead used local optimization to reduce processing time to search for the optimal multi-level thresholds. It is a divisive approach, which starts with a single class and recursively splits the single class into $\mathrm{M}$ classes. This algorithm can be described as following:

1. Start with a single class $C_{0}$ which has all intensity levels $t=0 \ldots 255$ and add to a list C with count $m=0$.

2. Compute total variance $\sigma_{0}^{2}$ in class $\mathrm{C}_{0}$ with equation 9 and the optimal threshold $\mathrm{t}$ with equation 10 .

3. Search each candidate class $C_{i}$ in list $C$ and find the class $c_{s}$, which has the largest total variance $\sigma_{i}^{2}$ and the optimal threshold $\mathrm{t}_{\mathrm{i}}$. Intensity gray level in class $\mathrm{C}_{\mathrm{i}}$ ranges from $\mathrm{t}_{\mathrm{s}}$ to $\mathrm{t}_{\mathrm{s}+1}$, where $0 \leq t_{1}<\ldots<t_{\mathrm{s}}<t_{s+1}<\ldots<L$

4. Split the class $C_{s}$ into two new classes $C_{m}$, and $C_{n}$. Intensity gray level in class $C_{m}$ ranges from $t_{s}$ to $t_{i}$ and ranges from $t_{i}+1$ to $t_{s+1}$ in class $c_{i}$.

5. Compute total variance $\sigma_{m}^{2}, \sigma_{n}^{2}$ and the optimal thresholds, $\mathrm{t}_{\mathrm{m}}$, and $\mathrm{t}_{\mathrm{n}}$ for two classes.

6. If any new created class $\mathrm{c}_{\mathrm{j}}$ has satisfied following criteria $\sigma_{j}^{2}<\sigma_{\min }^{2} \vee \operatorname{size}\left(c_{j}\right)<\min \_$size, classify this class as a none-divisible class. Otherwise this class is assigned as candidate class.

7. Remove $c_{s}$ from list $C$ and add two split classes $c_{m}$ and $c_{n}$ to list $C$. Increment $m$ by 1.

8. If the number of classes including all none-divisible classes and candidate classes has reached maximum threshold $\mathrm{M}$ or the largest variance is below some threshold, then stop, else go back to step 3 .

\subsection{Entropy-Based Thresholding}

The proposed entropy algorithm is another modification of [13] where the selection of optimal threshold in any class $\mathrm{C}_{\mathrm{i}}$ for $\left[\mathrm{t}_{\mathrm{i}-1}+1, \ldots, \mathrm{t}_{\mathrm{i}}\right]$ is defined as the maximum between-class entropy in equation 11:

$e_{B}=-\left\{\sum_{j=t_{i-1}}^{t}\left(\left(p_{j} / \omega_{1}\right) * \log _{2}\left(p_{j} / \omega_{1}\right)\right)+\sum_{j=t+1}^{t_{i}}\left(\left(p_{j} / \omega_{1}\right) * \log _{2}\left(p_{j} / \omega_{1}\right)\right)\right\}$.

where ${ }^{\omega_{1}=\sum_{j=t_{i-1}}^{t} p_{j}}, \omega_{2}=\sum_{j=t+1}^{t_{i}} p_{j}$.

\subsection{Peak-Seeking-Based Thresholding}

The selection of thresholds in [8] is to seek most significant peaks through several stages of peak detections and is described as the following steps:

1. Find all peaks.

2. Find significant peaks.

3. Remove any small peaks.

4. If two peaks are close, the peak with bigger value is chosen.

5. Remove a peak if the valley between two peaks is not obvious. 
6. The minimum values between any adjacent peaks are selected as the thresholds.

After thresholds of the histogram, F, in the first image are selected, every gray level, $i$, is labeled as one of the following:

$$
\begin{gathered}
L=\left\{l_{0,\left(0, t_{0}-1\right.}, l_{\left.1, t_{0}, t_{1}-1\right), \ldots,}, l_{j,\left(t_{j-1}, t_{j}-1\right.}, \ldots, l_{m,\left(t_{m}, t_{m}-1\right)}\right\}, \\
L_{F}(i)=l_{j,\left(t_{j-1}, t_{j}-1\right.}, \quad t_{j-1} \leq i \leq t_{j}-1,
\end{gathered}
$$

where $L_{f}(i)$ represents the $\mathrm{j}^{\text {th }}$ label value which gray level, $i$, is assigned to and $i$ is within the range $\left[\mathrm{t}_{\mathrm{j}-1}, \mathrm{t}_{\mathrm{j}}-1\right]$.

The process of assigning labels to gray levels in the second histogram, G, is a lookup operation, described as follows

$$
\left.L_{G}(j)=L_{F}(\text { lookupTbl[ } j]\right) \text {, }
$$

where lookupTbl represents mapping of $\mathrm{i}^{\text {th }}$, gray level in histogram $\mathrm{G}$ to $\mathrm{j}^{\text {th }}$ gray level in histogram $\mathrm{F}$ created in section 2.

Labeling each pixel in the images is another lookup operation which retrieves a label value through its gray level directly. Finally a connected component analysis is performed on both labeled images to extract regions where pixels have been assigned to the same value.

\section{Experiment}

To test performance of the proposed algorithm in histogram-based segmentation, various video sequences in Figure 2 are used in experiments. Each type has been further tested under varying scaling, rotation, and translation camera transformations.

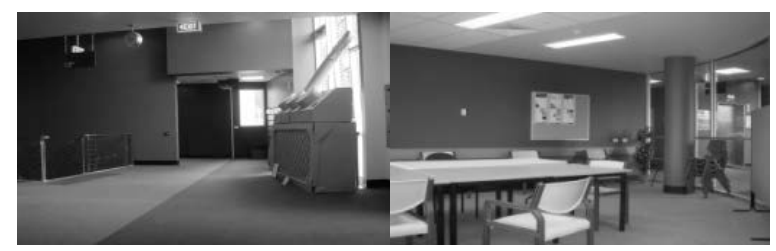

(a)

(b)

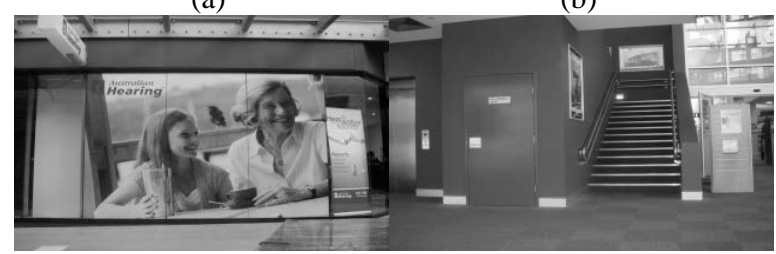

(c)

(d)

Figure 2. (a) Hall (b) Lounge (c) Ladies (d) Stairs

Testing images for rotation tests were selected every 40 frames from the input 
video sequences. The maximum frame interval range is 200 frames, approximating 10 degree's rotation which is reasonable in video sequences. Images in translation tests were selected every 20 frames among 100 frames as well. The horizontal translation was measured as percentage of amount of translation over the image width. The maximum translation range in the testing sequences was $15 \%$.

\subsection{Ground Truth Match Simulation}

We used exhaustive RANSAC method [13] to simulate the ground truth matches between region centroids. RANSAC is a non-deterministic algorithm for the estimation of a transformation model from observed data which contains outliers. It is essentially composed of two steps that are repeated iteratively.

Hypothesize: A sample of size $\mathrm{m}$ among the $\mathrm{N}$ data points is randomly selected. The model parameters are computed from this sample. $\mathrm{m}$ is the smallest sufficient cardinality to determine the model parameters.

Test: The hypothesis is verified against the rest of the data by counting the points consistent with the estimated model parameterization.

These two phases are repeated until the iteration finishes.

In video, 2D affine transformation can be modeled as composite matrix operation of scaling, rotation, and translation, which maps a point $\mathrm{p}=\left(\mathrm{x}_{\mathrm{i}}, \mathrm{y}_{\mathrm{i}}\right)^{\mathrm{T}}$ to a point $\mathrm{q}=$ $\left(\mathrm{u}_{\mathrm{i}}, \mathrm{v}_{\mathrm{i}}\right)^{\mathrm{T}}$ as follows:

$$
\begin{aligned}
{\left[\begin{array}{c}
u_{i} \\
v_{i} \\
1
\end{array}\right] } & =\left[\begin{array}{ccc}
1 & 0 & t_{x} \\
0 & 1 & t_{y} \\
0 & 0 & 1
\end{array}\right] *\left[\begin{array}{ccc}
\cos \theta & -\sin \theta & 0 \\
\sin \theta & \cos \theta & 0 \\
0 & 0 & 1
\end{array}\right] *\left[\begin{array}{ccc}
s & 0 & 0 \\
0 & s & 0 \\
0 & 0 & 1
\end{array}\right] *\left[\begin{array}{c}
x_{i} \\
y_{i} \\
1
\end{array}\right] \\
& =\left[\begin{array}{ccc}
\cos \theta * s & -\sin \theta * s & t_{x} \\
\sin \theta * s & \cos \theta * s & t_{y} \\
0 & 0 & 1
\end{array}\right] *\left[\begin{array}{c}
x_{i} \\
y_{i} \\
1
\end{array}\right]
\end{aligned}
$$

The affine matrix $\left[\begin{array}{ccc}\cos \theta * s & -\sin \theta * s & t_{x} \\ \sin \theta * s & \cos \theta * s & t_{y} \\ 0 & 0 & 1\end{array}\right]$, in equation 15 can be simplified as $M=\left[\begin{array}{ccc}a & -b & c \\ b & a & d \\ 0 & 0 & 1\end{array}\right]$ and the parameters in the simplified matrix can be solved by the pseudo-inverse solution. Therefore, the smallest sufficient cardinality to determine the model parameters in RASAC is two in this case.

Given a set of points and its correspondences in the form $\mathrm{U}=$ WA. 


$$
\left[\begin{array}{c}
u_{1} \\
\ldots \\
u_{n} \\
v_{1} \\
\cdots \\
v_{n}
\end{array}\right]=\left[\begin{array}{cccc}
x_{1} & -y_{1} & 1 & 0 \\
x_{1} & -y_{1} & 1 & 0 \\
y_{1} & x_{1} & 0 & 1 \\
& \ldots & & \\
y_{n} & x_{n} & 0 & 1
\end{array}\right] *\left[\begin{array}{l}
a \\
b \\
c \\
d
\end{array}\right]
$$

The pseudo-inverse solution $A=\left(W^{T} W\right)^{-1} W^{T} U$ is computed to solve for the four affine coefficients.

$\mathrm{U}$ and $\mathrm{M}$ are then used for calculating the re-projection error $e$ of the remaining pairs of correspondence. Re-projection error is defined as the Euclidean distance between the transformed point of point $\mathrm{p}$ in the previous frame according to the model $\mathrm{M}$ and the original correspondence of $\mathrm{q}$ in the current frame:

$$
e=\left|\left[\begin{array}{c}
x_{i}^{\prime} \\
y_{i}^{\prime} \\
1
\end{array}\right]-M\left[\begin{array}{c}
x_{i} \\
y_{i} \\
1
\end{array}\right]\right|
$$

The re-projection error threshold $T_{\text {thres }}$ is determined such that among all the initial matches, only those pairs with $e<T_{\text {thres }}$ will be regarded as inliers and kept. In our experiment, $T_{\text {thres }}$ is set to 2 . The process is repeated for different subsets, resulting in different transformations, with the number of inliers recorded for each transformation. The transformation supported by the highest number of inliers among all the iterations is selected to be the winning transformation. In the final step, all inliers from the winning transformation are considered to be the ground truth matches.

\subsection{Experiment Results}

We extracted the gravity centre points of each image region from two segmented images using both integrated-warped methods, and non-warped methods. In test cases of rotation, and translation, we found that both proposed warped method and nonwarped methods were able to provide similar number of matched regions because the change of the histogram is limited. Figure 3 shows number of ground truth matches found in segmented regions using integrated-warped method, and non-warped method for image Hall_r200 and Hall_r400 under 10’s degree rotation respectively.

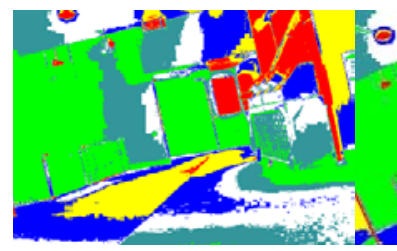

(a)

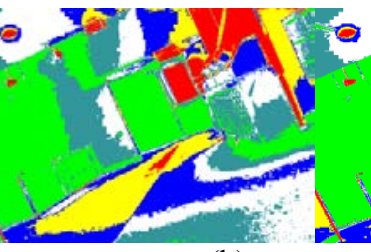

(b)

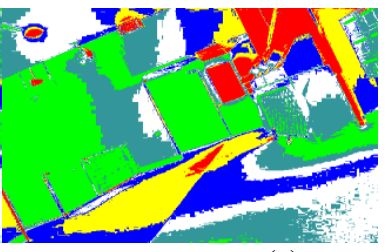

(c)

Figure 3. (a) segmented regions in Hall_r200 (b) warped segmented regions in Hall_r400 (c) non-warped segmented regions in Hall_r400 
Table 2: Matching Results for Scaling

\begin{tabular}{|c|c|c|c|c|c|c|}
\hline & \multicolumn{3}{|c|}{ Ladies } & \multicolumn{3}{|c|}{ Lounge } \\
\hline & integ & $d p$ & non-warped & integ & $d p$ & non-warped \\
\hline \multicolumn{7}{|c|}{ scaling ratio 1.3} \\
\hline Peak-Seeking-Based & 21 & 24 & 20 & 17 & 20 & 10 \\
\hline Entropy-Based & 28 & 30 & 22 & 18 & 21 & 9 \\
\hline Clustering-Based & 30 & 31 & 24 & 15 & 23 & 13 \\
\hline \multicolumn{7}{|c|}{ scaling ratio 1.4} \\
\hline Peak-Seeking-Based & 19 & 23 & 12 & 15 & 20 & 6 \\
\hline Entropy-Based & 29 & 28 & 15 & 17 & 18 & 10 \\
\hline Clustering-Based & 27 & 28 & 25 & 17 & 20 & 13 \\
\hline & \multicolumn{3}{|c|}{ Hall } & \multicolumn{3}{|c|}{ Stair } \\
\hline \multicolumn{7}{|c|}{ scaling ratio 1.3} \\
\hline Peak-Seeking-Based & 21 & 20 & 12 & 12 & 12 & 7 \\
\hline Entropy-Based & 17 & 16 & 17 & 10 & 13 & 10 \\
\hline Clustering-Based & 20 & 19 & 16 & 10 & 7 & 10 \\
\hline \multicolumn{7}{|c|}{ scaling ratio 1.4} \\
\hline Peak-Seeking-Based & 18 & 19 & 9 & 11 & 10 & 4 \\
\hline Entropy-Based & 17 & 16 & 15 & 10 & 11 & 10 \\
\hline Clustering-Based & 18 & 20 & 11 & 10 & 7 & 11 \\
\hline
\end{tabular}

In test cases of scaling, we selected test images with zooming ratio up to 1.4. Table 2 shows the number of matched regions for Hall sequence under 1.3 and 1.4 scaling. The warping methods performed better than non-warped histogram-based methods $95 \%$ of the time. And the warping methods found more number of matches on average $40 \%$ higher than those non-warped methods. In these experiments, the DPbased DTW performed only 6\% better than the integration-based method as shown in Table 2. Experiments results in Figure 4 show matched regions using DP-warped method, integration warped method, and non-warped method for image Hall_1 and Hall_4 with 1.4 scaling respectively.
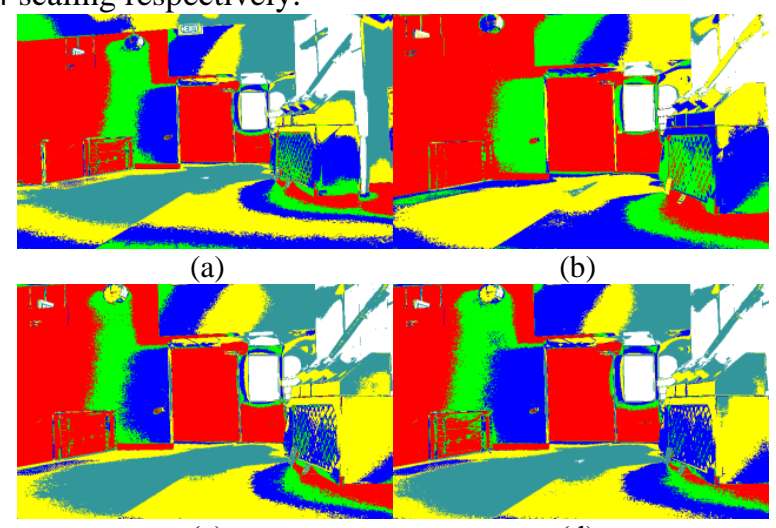

(c)

(d)

Figure 4. (a) segmented regions in Hall_1 (b) non-warped segmened regions in Hall_4 (c) dpwarped segmented regions in Hall_4 (d) integ-warped segmented regions in Hall_4 


\section{Conclusion}

This paper has presented methods for improving histogram-based segmentation consistency in video sequences for image sequence registration using a DTW approach. The use of DTW is able to improve segmentation consistency by up to $40 \%$. This is necessary for making histogram segmentation based image sequence registration robust against image scaling. While the classical DP-based DTW marginally outperforms the proposed integration-based approach, it is about 430 times slower. Designed for low computation requirements, the proposed technique is effectively able to handle image sequences with significant histogram changes due to either scaling or lighting changes. 


\section{References}

[1] L.G.Brown, “A survey of image registration techniques,” ACM Computing Surveys 24(4), pp.325-376, December 1992.

[2] B. Zitová, Jan Flusser: "Image registration methods: a survey.” Image Vision Comput. 21(11): 977-1000 (2003).

[3] J. B. A. Maintz and M. A. Viergever, "A survey of medical image registration,” Medical Image Analysis 2(1), 1998.

[4] J.Flusser, T. Suk, "A Moment-based Approach to Registration of Images with affine Geometric Distortion.”, IEEE Trans on Geoscience and Remote Sensing, Vol 32. No.2 March, 1994.

[5] X. Dai and S. Khorram, "A feature-based image registration algorithm using improved chain-code representation combined with invariant moments," IEEE Trans. Geosci. Remote Sensing, vol.37, Sept. 1999.

[6] A. Goshtasby, G. C. Stockman, and C. V. Page, "A region-based approach to digital image registration with subpixel accuracy,” IEEE Trans. Geosci. Remote Sensing, vol. 24, no. 3, pp. 390-399, 1986

[7] J. S. Weszka, R. N. Nagel, and A. Rosenfeld, “A threshold selection technique”, IEEE Trans. Comput., vol. C-23, pp. 1322-1326, 1974.

[8] H. D. Cheng and Y. Sun, "A hierarchical approach to color image segmentation using homogeneity,” IEEE Trans. Image Process., vol. 9, no.12, pp. 2071-2082, Dec. 2000.

[9] N. Otsu, "A threshold selection method from gray-level histograms," IEEE Transactions on Systems Man Cybernet SMC-9, 1979, pp. 62-66.

[10] J.N. Kapur, P.K. Sahoo, A.K.C. Wong, "A New Method for Gray-Level Picture Thresholding Using the Entropy of the Histogram," Graphical Models and Image Processing, 29, 273-285, 1985.

[11] H. Gonçalves, J. A. Gonçalves, and L. Corte-Real, "HAIRIS: A method for automatic image registration through histogram-based image segmentation,” IEEE Trans. Image Process., vol. 20, no. 3, pp. 776-789, 2011.

[12] Chu, S., E. Keogh, D. Hart \& Michael Pazzani. "Iterative Deepening Dynamic Time Warping for Time Series." In Proc. of the Second SIAM Intl. Conf. on Data Mining.Arlington, Virginia, 2002.

[13] Martin A. Fischler and Robert C. Bolles. "Random Sample Consensus: A Paradigm for Model Fitting with Applications to Image Analysis and Automated Cartography". Comm. of the ACM 24 (6): 381-395, 1981. 